\title{
STRUCTURAL ANALYSES OF GLARE-GFRP TRANSITION FOR INTEGRATED VHF ANTENNA ON A FUSELAGE PANEL
}

\section{EMUS 2020}

\author{
F. TURON ${ }^{*}$, F. OTERO ${ }^{*}$ AND X. MARTINEZ ${ }^{* \dagger}$ \\ International Center for Numerical Methods in Engineering (CIMNE) \\ Universidad Politécnica de Cataluña \\ Campus Norte UPC, 08034 Barcelona, Spain \\ e-mail: congress@cimne.upc.edu,www.cimne.com \\ $\dagger$ Departamento de Ciencia e Ingenieria Nautica (FNB) \\ Technical University of Catalonia (UPC) \\ Pla de Palau 18, 08003 Barcelona, Spain \\ email: semni@cimne.upc.edu,www.semni.org
}

Key words: Smart fuselage panel, composite materials, serial-parallel mixing theory, constitutive modelling, finite element methods

\begin{abstract}
The objective of this paper is to describe the numerical simulation of the transition zones in the multifunctional fuselage panel design to integrate a VHF (Very High Frequency) antenna. The panel is made of GLARE (Glass Laminate Aluminium Reinforced Epoxy) with a central window of GFRP (Glass Fibre Reinforced Polymer). The structural analyses of the transition GLARE/GFRP is presented along with its experimental validation. The numerical simulations showed a very high level of correlation with the experimental tests, both in the longitudinal load vs displacement curves and deformations captured with strain gauges and DIC (Digital Image Correlations).
\end{abstract}

\section{INTRODUCTION}

Providing composite structures with new functionalities often involve merging different material configurations, including layups and components, into a single continuous structure. This can produce transition zones with complex interactions between layers that must be studied in detail by simulations. Moreover, numerical simulations can also assist in the design process of this new composites structures.

This approach is put in practice during the procedure exposed on this paper, from the initial phase of assisted design to the experimental verification. The resultant GLARE/GFRP transition archived is broadly analysed with both, an experimental campaign and its equivalent numerical analysis. Most relevant results for the two representative orientations of final layout are presented. These are, the optimal and more demanded layout properly oriented i.e. Section BB in Figure 1, which is referred as MST-B (Mechanical Static Test) and secondly its complementary orientation MST-C in which the fibres are rotated 90 degrees. This second configuration is placed in less demanded direction. 

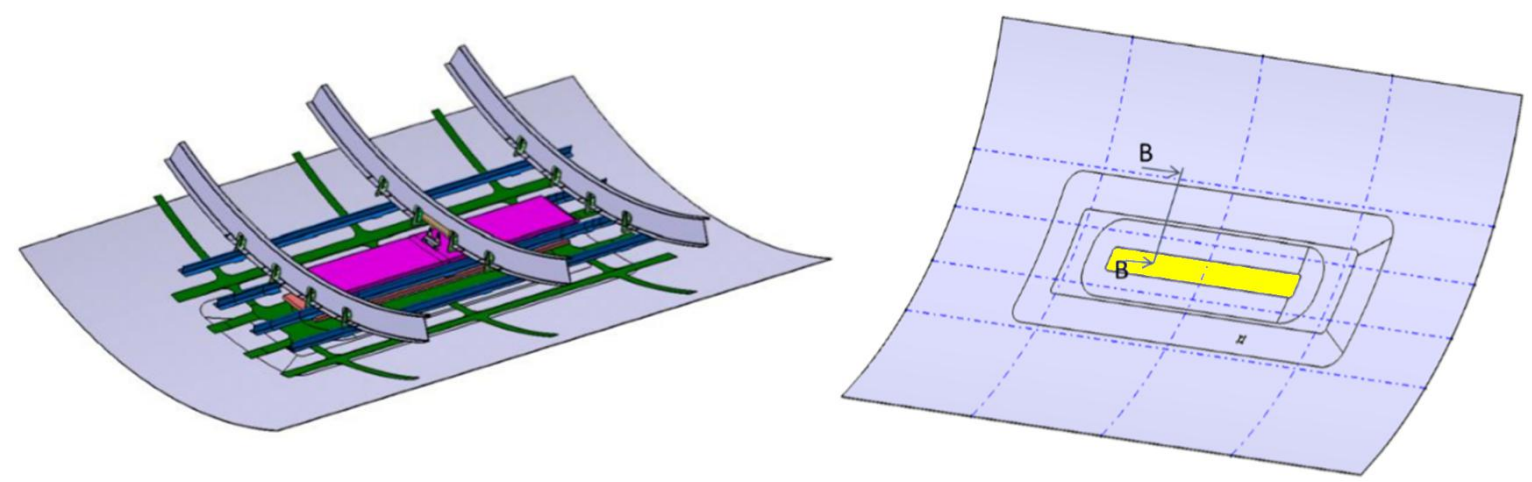

Figure 1: Integrated VHF Antenna in fuselage structure.

\section{Design procedure.}

The design procedure carried out to find the best transition to join the GLARE laminate with the GFRP windows located at the centre of the fuselage panel (shown in yellow at Figure 1) begun with the numerical simulation of a predesign. The procedure shows some critical spots between the end of the aluminium sheets, proceeding from the GLARE laminate, and the beginning of the GFRP layers replacing them.

A better response was achieved when orientating the fibres of these GFRP layers following (parallel) the edge of the aluminium sheets as shown in Figu of the glass caused a sud that resulted in a quick being structurally critical, the prevention of long-term performance.
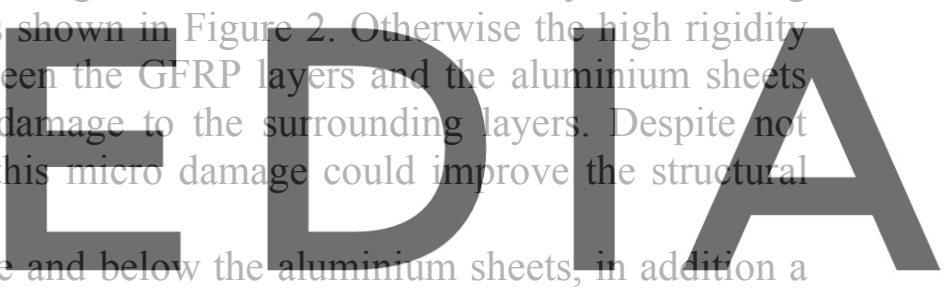

small resin gap is added between the parallel GFRP and the aluminium layers next to them

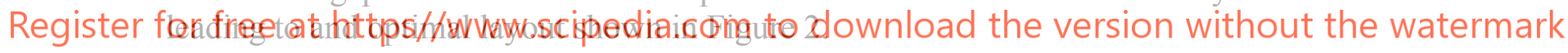

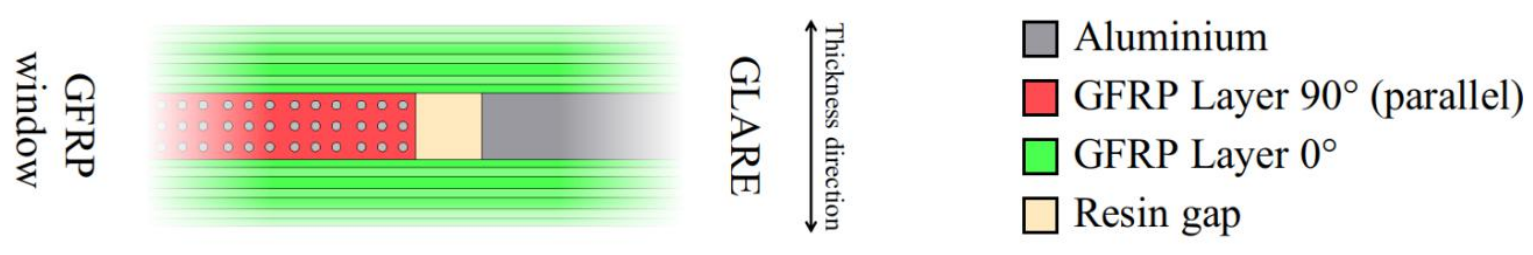

Figure 2: Critical spot from optimal orientation layout configuration for transition cross section BB.

In this way the stresses of the aluminium sheets are transferred to the surrounding long fibres across its whole upper and lower contact surfaces and the epoxy matrix of the parallel layers benefits from the high stiffness and low deformation of these. The resulting transition geometry manage to stream the stresses between its ends gradually, minimizing the areas of stress concentrations. Therefore, reducing the critical points that can lead to intralaminar fracture and delamination phenomena. The whole final layup of the transition are confidential and can not be shown on this publication. 


\subsection{GEOMETRY AND TEST SETUP OF THE SPECIMEN}

The dimensions of the tested specimens are $700 \mathrm{~mm}$ length and $100 \mathrm{~mm}$ width, the free length between jaws is $580 \mathrm{~mm}$ (See Figure 3). In Figure 3 the location of the strain gauges i.e. 1 and 3 on the inner side and 2 and 4 on the outer side (see red cross points) are shown.
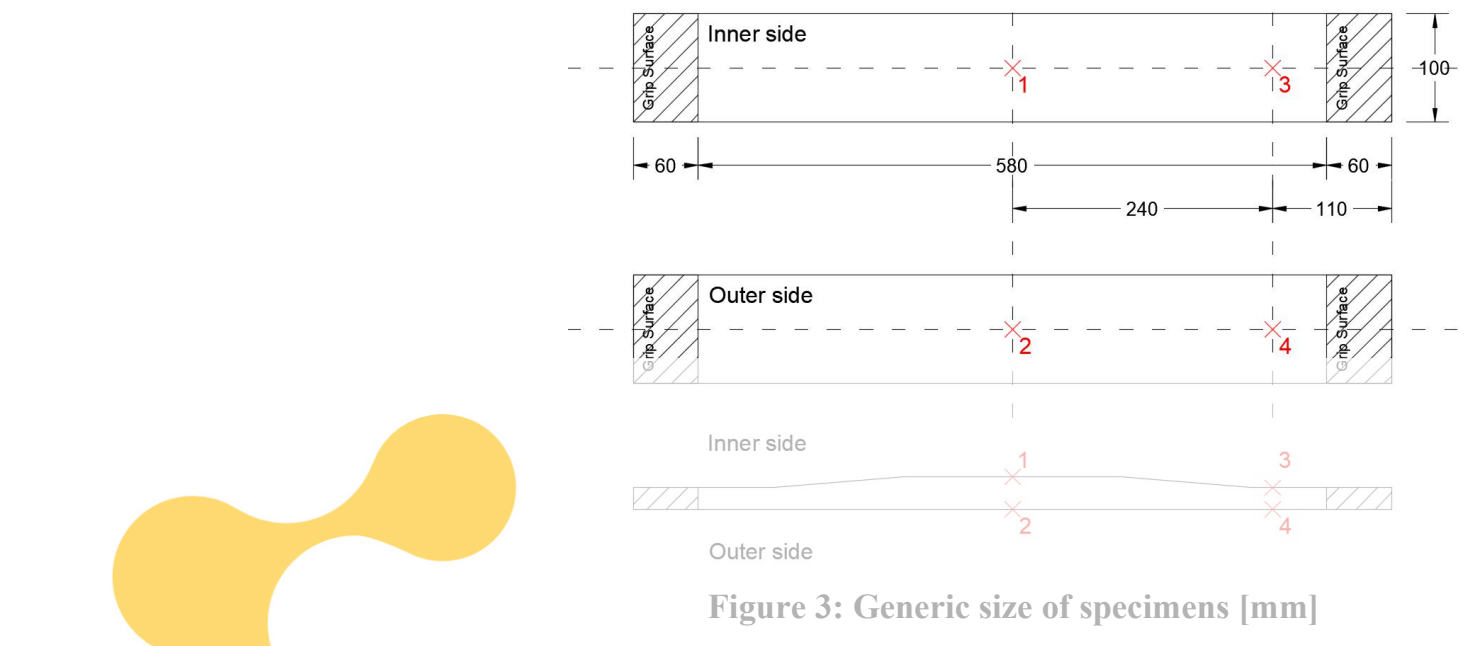

In the experimental test, a displacement is imposed by means of a piston that slowly displaces one jaw mean while the other remains steady. The reaction of the specimen and the
imposed displacement are recorded. Moreover, deformations are moasured using the four
strain gauges together with a Digital Inage Corretation (DIC) equipment.
1.2 NUMERICAL MODEL

The numerical model developed reproduces the geometry of the laminate layer by layer

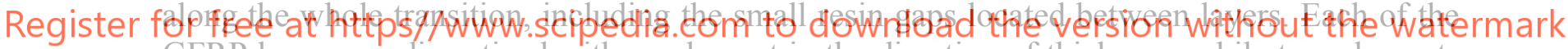
GFRP layers are discretized with an element in the direction of thickness, while two elements are used in the aluminium sheets. Then, it allows to analyse the stress-strain state of each layer individually. The finite element mesh has approximately 250.000 hexahedral linear elements with 8 nodes and 8 Gauss points.

GFRP is simulated using the mixing theory Serial-Parallel [1][2][3] already used in the analysis of a multifunctional Orthogrid Panel [4]. It together with the non-linear constitutive models used in the the fibres and matrix, it is able to monitor the onset and evolution of damage and plasticity, On the other hand, the aluminium material is characterized with a plastic constitutive law, which reproduces accurately the non-linear behaviour of GLARE.

The numerical simulation takes advantage of the symmetry of the specimen and its layout and only a quarter of the total geometry is simulated. The effect of the clamp is implemented by imposing a displacement in the $\mathrm{Y}$ direction and restricting movement also in the $\mathrm{X}$ direction on all nodes under the grip area.

Experimentally it was proved that the displacements out of the plane were low and their impact could be neglected. For these reasons the analysis were made within the small deformations paradigm, but in order to prevent fictitious bending was necessary restrict movement in the $\mathrm{Z}$ direction on the entire outer face of the panel. 


\section{NUMERICAL RESULTS}

In the present section the comparison of the numerical vs experimental results is presented for the two configuration studied. The loads and displacement from the piston test machine, as well as the strains measured with the strain gauges and captured with the DIC are used for the comparison.

\subsection{LAYOUT CONFIGURATION MST-B}

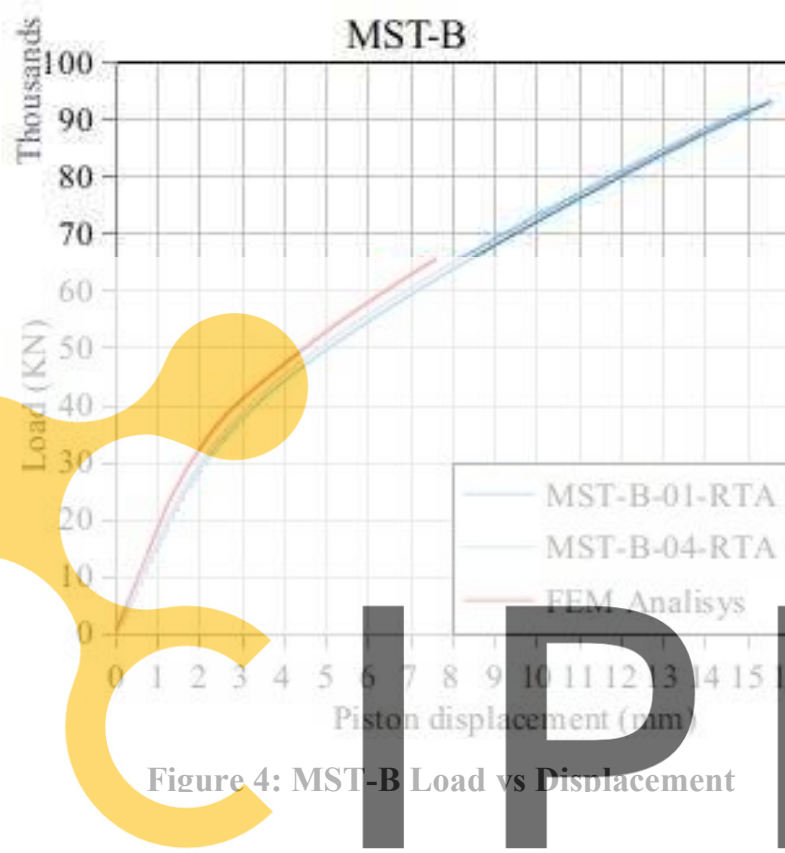

Figure 4 shows the load vs displacement curve for configuration MST-B.

The initial stiffness obtained in the experimental test is very similar in comparison with the numerical analysis. When one-millimetre displacement is reached, the experimental results show a progressive loss of stiffness. The numerical simulation also shows accurately this stiffness loss. However the overall stiffness is slightly higher.

The load vs strain curves obtained with the strain gauges and DIC show a good
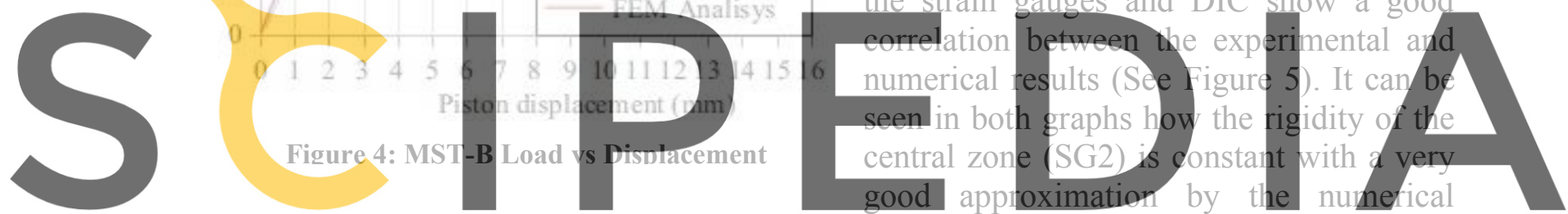

analysis. The response in the GLARE is not that high and it suffers a loss of stiffness between

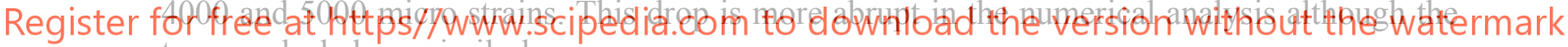
two graphs behave similarly.
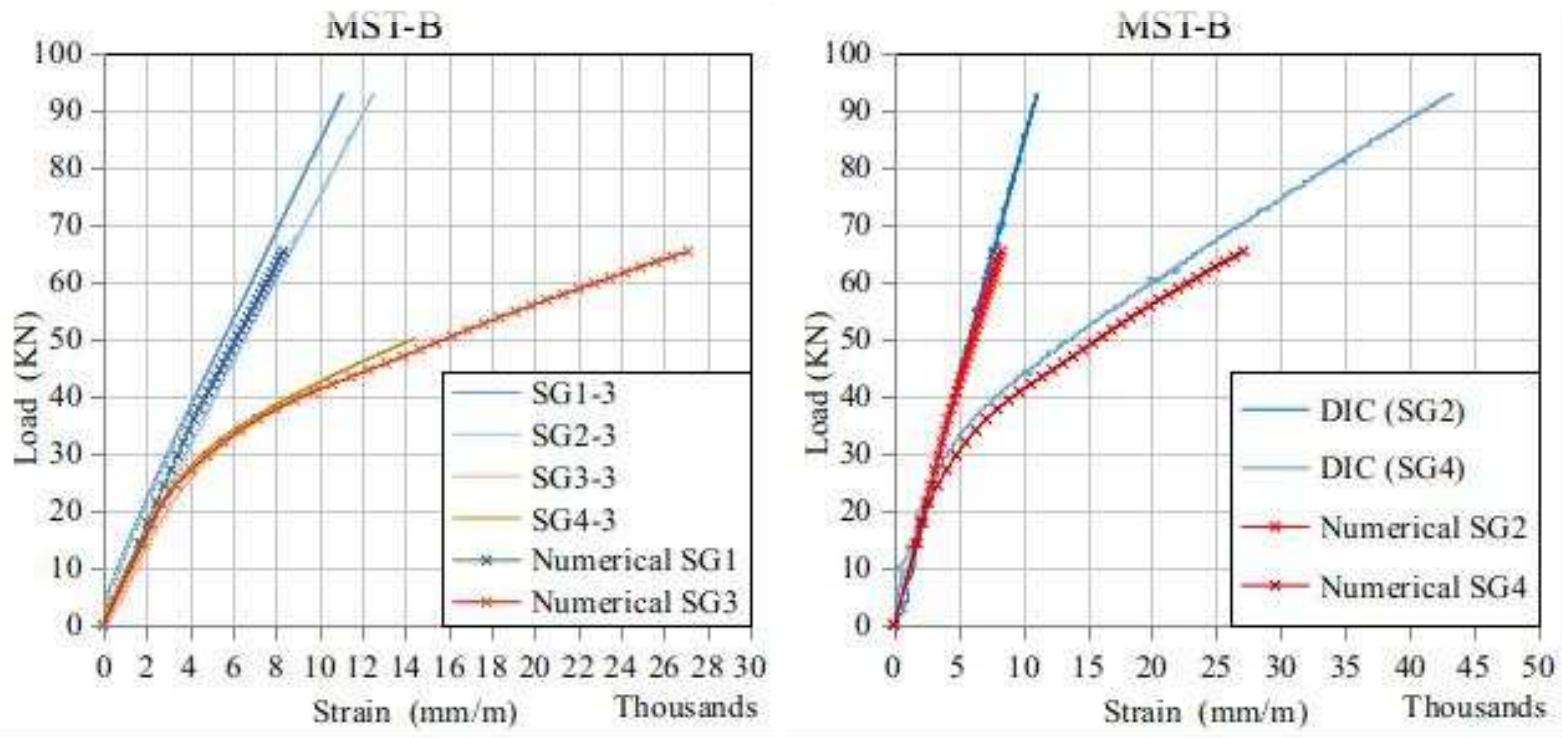

Figure 5: Resultant Load vs Strain curves of B configuration (stain gauges - DIC) 


\subsection{LAYOUT CONFIGURATION MST-C}

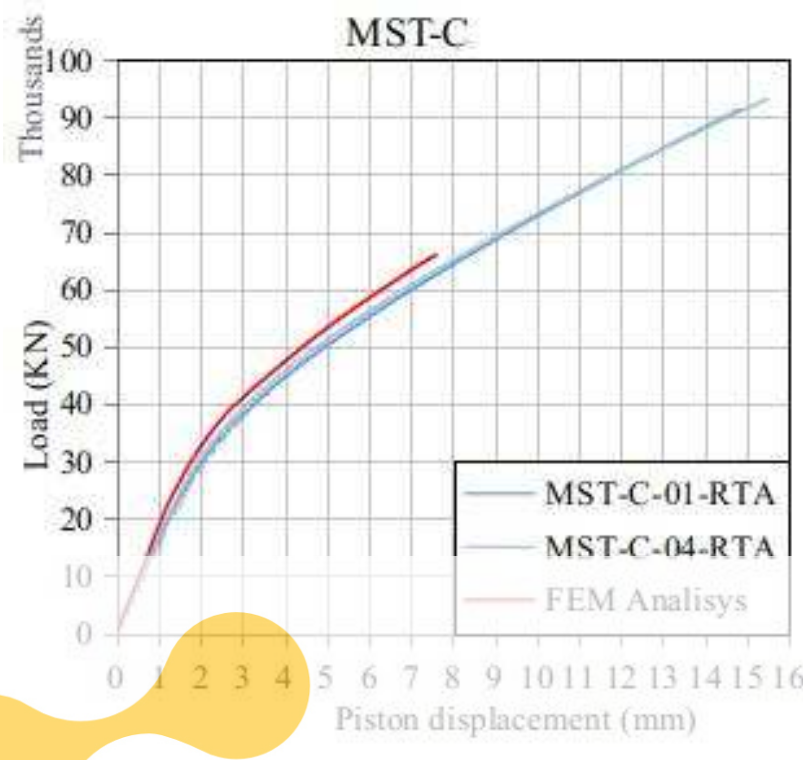

Figure 6: MST-B Load vs Displacement

Despite being poorly oriented the experimental overall response of configuration MST-C is almost identical to that measured in configuration MST-B. In addition the numerical analysis shows a extremely similar behaviour (see Figure 6).

The numerical analysis also shows a good correlation with the deformation recorded in the central strain gauge as well as in the GLARE sections as can be seen in Figure 7.

However, due to the non-optimal orientation of the fibres adjacent to the aluminium sheets, more and more damage zones are registered in the resin that joins them, as well as in the resin of the perpendicular GFRP layers located

immediately above and below
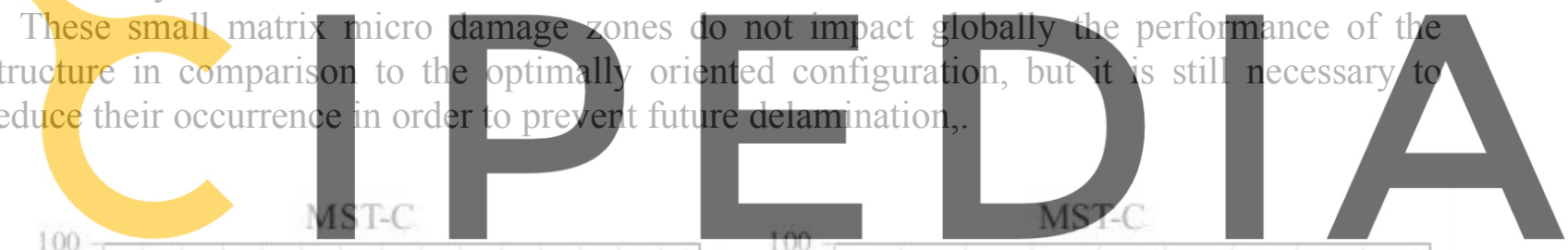

Register for free at https//www.scipedia.com to download the version without the watermark
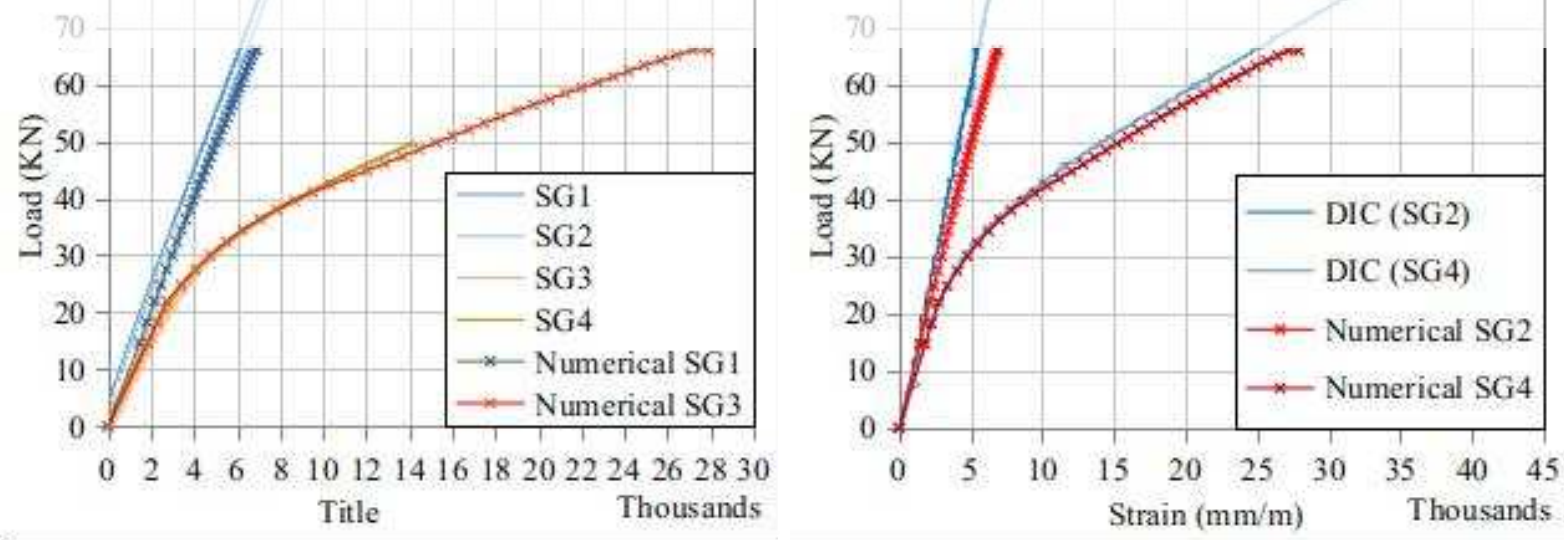

Figure 7: Load vs strain of Test MST, for Layout C 


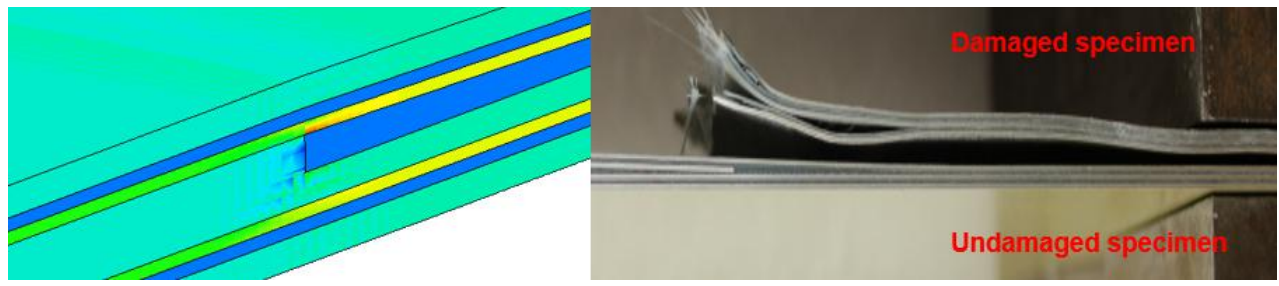

Figure 8: Breakage area

Finally, it can be observed in Figure 8 how the breakage zone in the experimental test coincides with the zone with the highest stresses found in both numerical simulation, configuration $\mathrm{B}$ and $\mathrm{C}$. In this area, the stresses in the fibres orientated in longitudinal direction reach the ultimate tensile strength causing the overall failure of the sample.

\section{CONCIUSIONS AND FINAI REMARKS}

- The numerical simulation shows a predicted initial stiffness in good agreement with the experimental results. The global response of both configurations tested starts with a nonlinear behaviour around $0.8 \mathrm{~mm}$ of piston displacement as it can be observed in the load vs displacement experimental curves (see Figures 4 and 6). The numerical results follow a very similar behaviour but with a slightly higher stiffness. After that, both curves, i.e. experimental and numerical, remain parallel with the same slope.
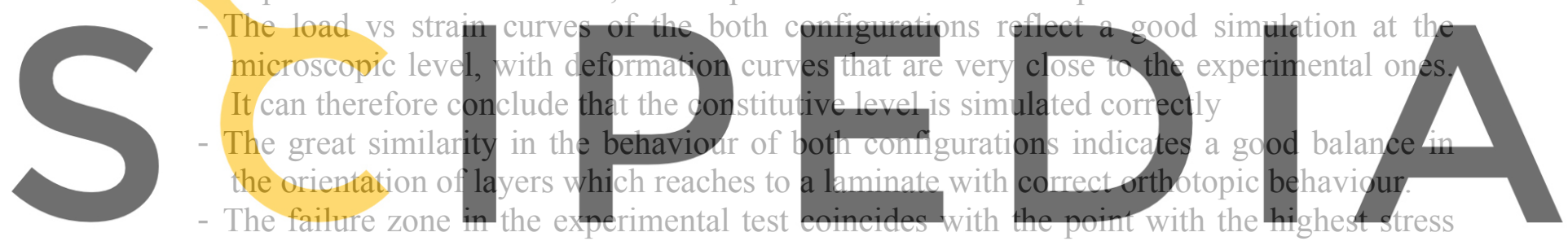

concentration in the numerical simulations. The strain recorded just before the break

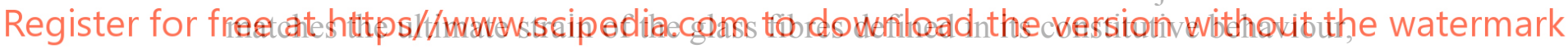

\section{REFERENCES}

[1] Rastellini F. and Oller S. and Salomón O. and Oñate, E. Composite materials non-linear modelling for long fibre-reinforced laminates continuum basis, computational aspect and validations. Computers and Structures. (2008) 86:879-896.

[2] Car E. and Oller S. and Oñate, E. An anisotropic elastoplastic constitutive model for large strain analysis of fiber reinforced composite materials. Computer Methods in Applied Mechanics and Engineering. (2000) 185:245-277.

[3] Martinez X. and Oller S. Numerical simulation of matrix reinforced composite materials subjected to compression loads. Archives of computational methods in engineering. (2009) 16:357-397.

[4] Francesc Turon, Fermin Otero And Xavier Martinez. Structural Analyses Of Orthogrid Fuselage Panel For Integrated Ku-Band Satcom Antenna - Emus 2019

Acknowledgements: This work has been supported by the European Union's Horizon 2020 research and innovation programme under grant agreement No. 723167 (ACASIAS project). 